\title{
The Place of Gold in the Nano World
}

\author{
Gareth M Francis and Richard E Palmer \\ Nanoscale Physics Research Laboratory, School of Physics and Space Research, \\ University of Birmingham, \\ Edgbaston, Birmingham B15 2TT, UK
}

Nanotechnology is the application of science on the nanometre scale. It is a generic technology, with potential applications in a wide range of sectors, from electronics and computing to sensors and catalysis. In this article we ask the question, 'does gold have a role in the nanoscale world?' We have investigated the growth of three dimensional gold nanoclusters on a graphite substrate by means of scanning tunnelling microscopy (STM). The first stages of the growth of nanoscale gold wires have been demonstrated, via the nucleation of gold clusters along steps of the surface. We also show that small clusters which are approximately 1-3nm in diameter are grown on the flat terraces, and demonstrate the movement of larger clusters across the surface with the STM tip. Prospects for the application of these nanoscale building blocks are discussed.

\section{ATOMIC CLUSTERS}

Atomic clusters represent new building blocks for the fabrication of nanostructured materials and films. Clusters are of particular interest because they have properties which lie between the atomic regime and the bulk (condensed matter) states. Their electronic, optical, chemical and physical properties change with their size, due to the confinement of electrons in a small volume (1), and generally tend towards the properties of the solid state at very large sizes. For example, the transition from an electrically insulating to metallic state has been found to occur at a size of approximately 100 atoms for gold clusters (2).

Gold clusters, like silver, copper and alkali metal clusters, exhibit especially marked size-dependent properties. Specifically, they show 'magic numbers', meaning that clusters composed of certain numbers of atoms are particularly stable. These stable clusters have closed shells of electrons (i.e. 2, 8, 20, 40... electrons), where each atom contributes one electron (3). The stability and hence ionisation energy of gold clusters also depends on whether the atom has an odd or even number of electrons, even numbers being more stable (4).

A beam of clusters, e.g. of gold atoms, can be produced by several methods. Chipping the clusters from a bulk solid by sputtering techniques and the condensation of vaporized atoms in an inert carrier gas are two examples. Clusters produced in a beam can also be deposited onto a substrate. In addition, the growth of gold and other noble metal clusters on surfaces via atomic vapour deposition in vacuum has gathered much interest over the last 15 years, especially with the development of atomically resolving spatial imaging techniques such as high resolution transmission electron microscopy (5) and scanning tunnelling microscopy (STM) (6) (The STM uses quantum mechanical electron tunnelling between a sharpened metallic tip and a surface, to image the contours of the surface electron density). Moreover, gold clusters have been deposited (7) on surfaces by applying a voltage pulse to an STM tip made of, or coated with, gold and placed a few nanometres above the surface. In addition, self-assembled molecular nanostructures have been constructed of gold clusters and thin organic films (8). Interest in metal clusters on surfaces is also motivated by heterogeneous catalysis, since clusters have a large surface area to volume ratio, e.g. gold cluster catalysts have been produced for oxidation reactions (9).

\section{IMAGING GOLD CLUSTERS ON A SURFACE}

We have produced gold clusters on a surface by depositing gold atoms onto the relatively unreactive surface of graphite (highly oriented pyrolytic graphite, 
HOPG) in high vacuum. Due to the weak interaction between the adsorbate and substrate, the gold grows in small 2 and 3 dimensional clusters (Volmer Weber growth). Deposited gold atoms diffuse across the flat surface at room temperature with little hindrance, and their behaviour is mainly governed by the presence of other gold atoms and by reactive surface steps or defects.

We have employed the scanning tunnelling microscope (STM) to investigate the growth of gold clusters on graphite and their mobility on the surface. The picture which emerges from these studies, and similar investigations of the growth of silver clusters (10) on the same surface, is as follows. When gold atoms collide with one another on the flat terrace a critical nucleus is formed. This acts as a sink for other atoms which will attach to it, forming a cluster. Graphite (HOPG) can be cleaved easily to form a flat surface with very few defects. However, steps are formed on the surface with average separations of -1 micrometre. Gold atoms which reach a step stick irreversibly to it and are then mobile only along the step edge (at room temperature) - like beads threaded on a string. In this environment atoms collide to form clusters and quasi-one-dimensional chains of clusters are produced.

Figure 1 shows a large area scan of the surface of graphite with small gold clusters decorating both the terraces (flat regions) and the surface steps. Figure 2 shows a close up of a terrace region randomly decorated with clusters. These clusters are approximately $1-3 \mathrm{~nm}\left(1\right.$ nanometre $\left.(\mathrm{nm})=1 \times 10^{-9} \mathrm{~m}\right)$ in diameter and are unperturbed by the scanning tip of the STM (see below). Figure 3 shows a surface step decorated with clusters. In fact, we have found that it is quite difficult to image clusters at the step with the STM, and we believe this is because the clusters can be moved from the steps by the scanning STM tip. Figure 4 shows a detailed STM image of gold clusters on the graphite surface; individual atoms in the substrate and the detailed shape of the clusters can be resolved.

Prospects for nanoscale fabrication with gold clusters are illustrated by our recent scanning electron microscopy (SEM) studies of silver clusters $(10,11)$ on the graphite surface. Silver clusters are preferentially formed at the steps, and by increasing the substrate temperature the probability of atomic collisions on the terrace can be decreased to the extent that clusters grow only at the steps, forming prototypical nanoscale 'wires' (Figure 5). Similar behaviour is expected of gold. We also found evidence that silver clusters are mobile on the terraces at room temperature (10) and

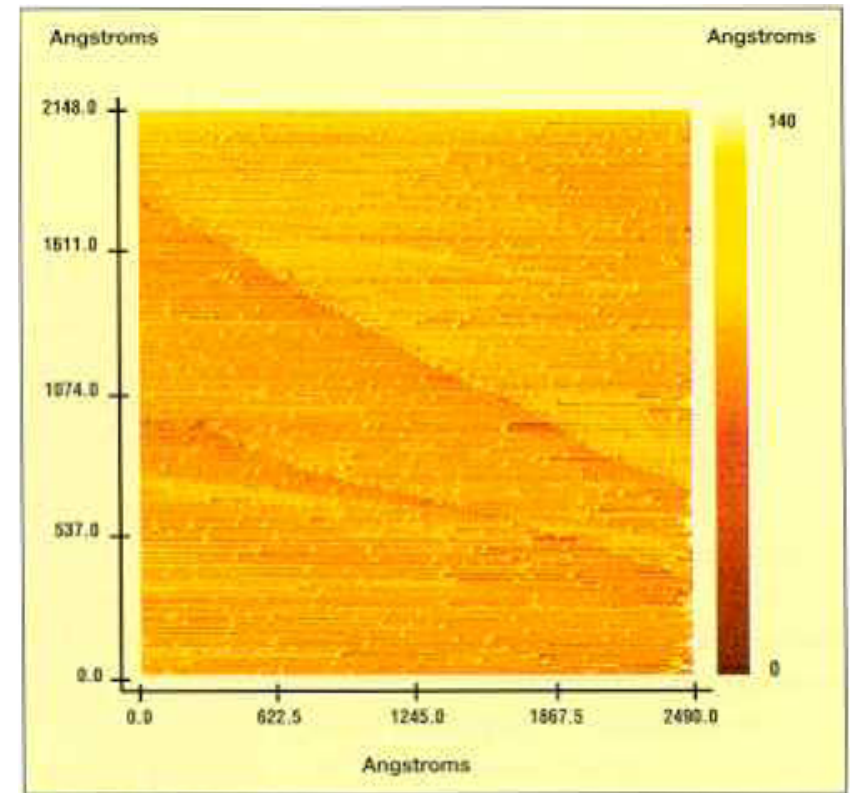

Figure 1 STM image of graphite $(2148 \times 2490 \AA)$ after deposition of 0.2 monolayers $A$ u over 20 sec at $20^{\circ} \mathrm{C}$ showing gold clusters decorating the terraces and a step (tunnelling current $I=2 \mathrm{nA}$, sample bias $=+0.2 \mathrm{mV}$ ).

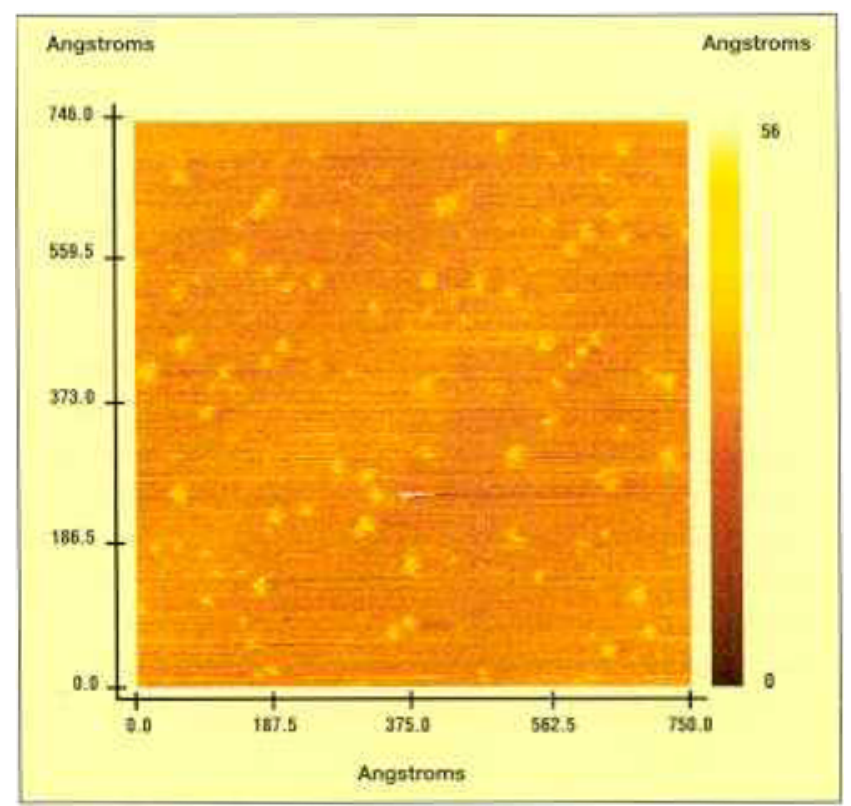

Figure $2 S T M$ image of graphite $(746 \times 750$ A) after deposition of 0.2 monolayers $A u$ over $20 \sec$ at $20^{\circ} \mathrm{C}$ showing a random array of $1-3 \mathrm{~nm}$ gold clusters (tunnelling current $I=2 \mathrm{nA}$, sample bias $=+0.2 \mathrm{mV}$ ). 


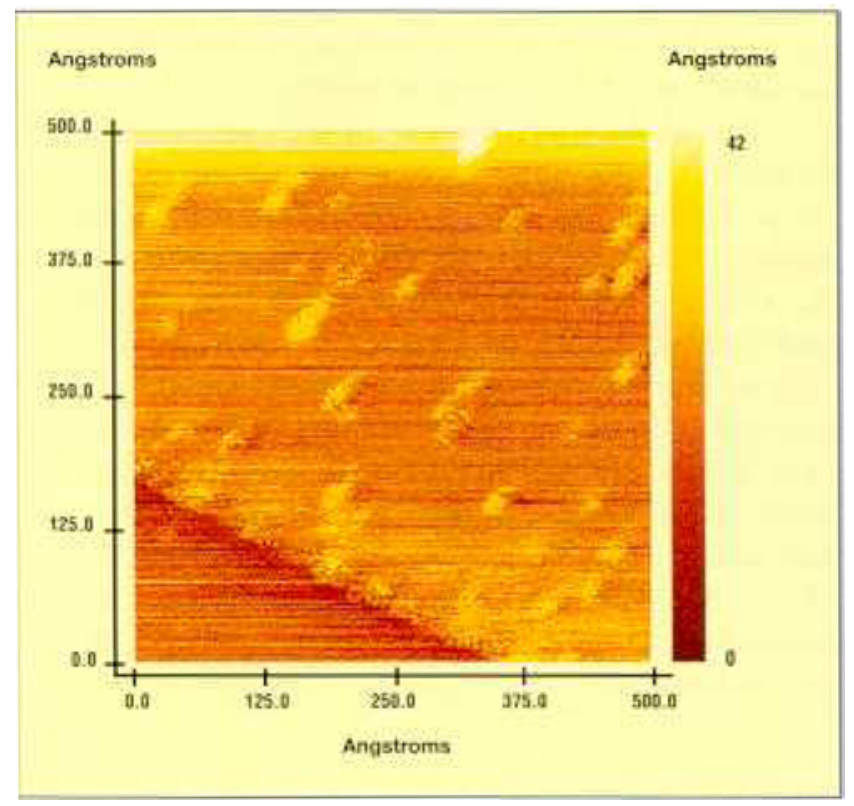

Figure 3 STM image of graphite $(500 \times 500 \AA)$ after deposition of 0.2 monolayers $A u$ over $20 \mathrm{sec}$ at $20^{\circ} \mathrm{C}$ showing a surface step decorated by gold clusters (tunnelling current $I=2 \mathrm{nA}$, sample bias $=+0.2 \mathrm{mV}$ ).

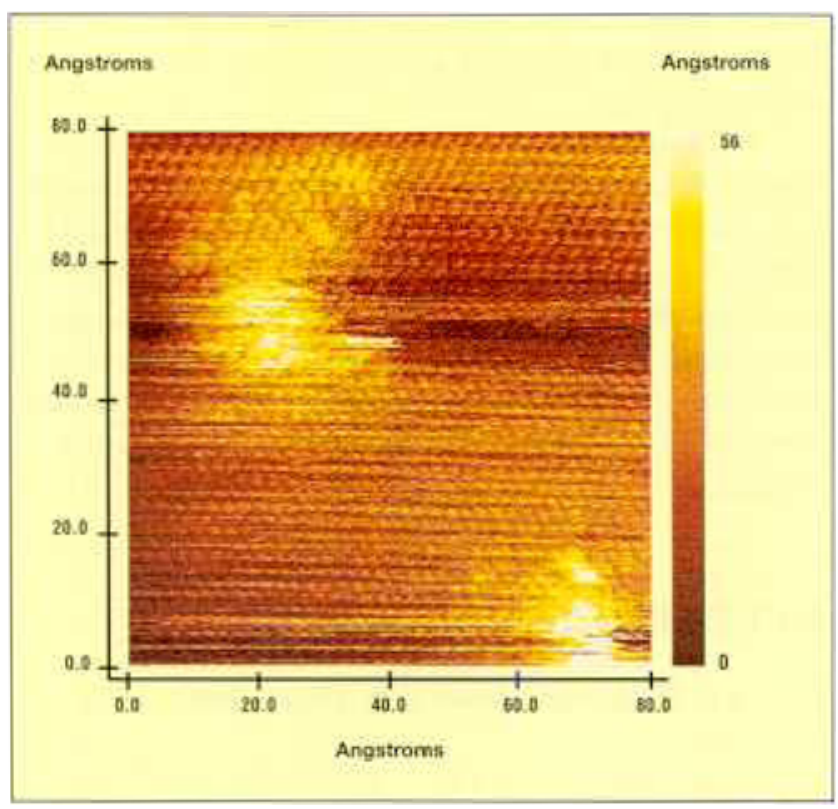

Figure 4 STM image of graphite $(80 \times 80 \AA)$ after deposition of 0.2 monolayers $A$ u over $20 \mathrm{sec}$ at $20^{\circ} \mathrm{C}$ showing two gold clusters, each with diameter $-1.5 \mathrm{~nm}$, and the surrounding substrate lattice (tunnelling current $I=2 \mathrm{nA}$, sample bias $=+0.2 \mathrm{mV}$ ).

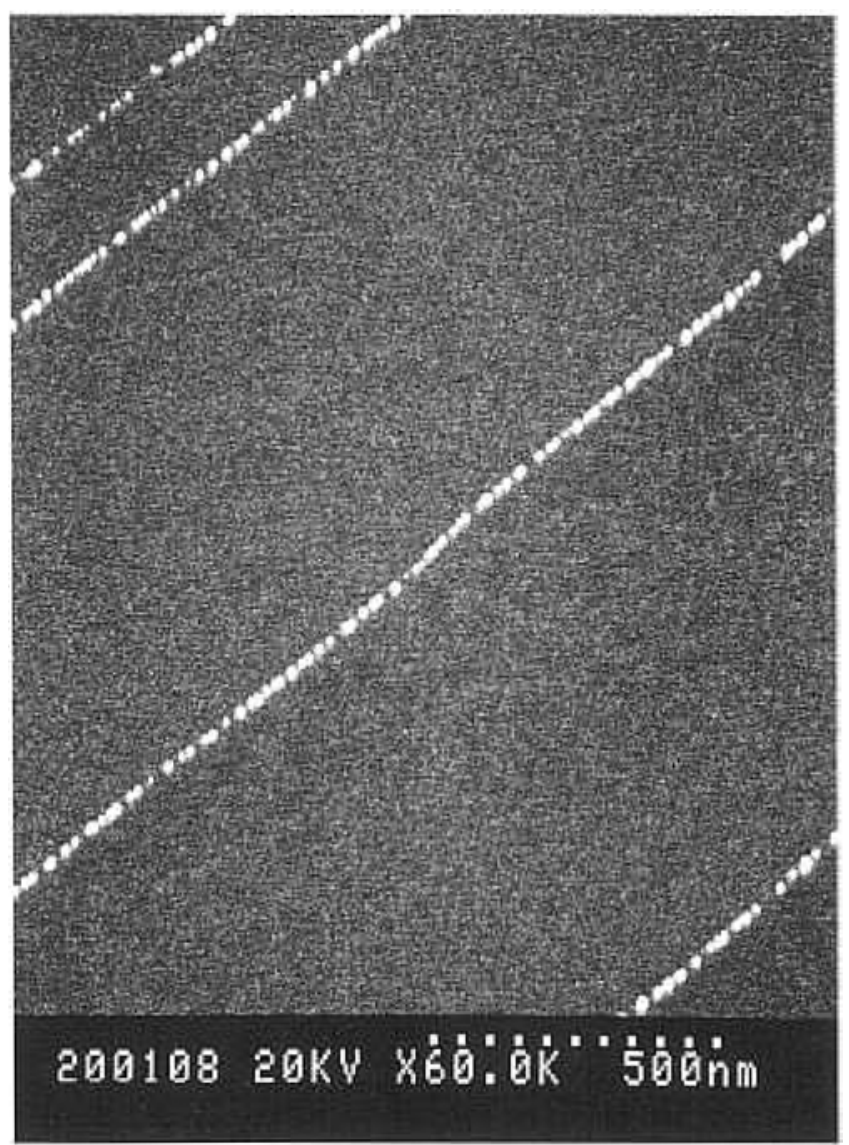

Figure 5 SEM image of graphite $(1750 \times 1450 \mathrm{~nm})$ after deposition of 0.01 monolayers of silver over 1 sec at $100^{\circ} \mathrm{C}$ showing prototypical nanoscale "wires", made from clusters grown at the surface steps.

stabilize themselves by grouping together. Indeed, at higher temperatures $\left(>100^{\circ} \mathrm{C}\right)$ the clusters grown at the steps are also mobile along the steps, and some coalescence occurs as a result.

SEM studies show some intriguing differences from the STM images. In particular the SEM tends to show predominantly larger clusters $(8-10 \mathrm{~nm})$ than those typically observed in the STM images $(1-3 \mathrm{~nm}$ in diameter). A possible reason for this is that the larger clusters are less strongly bonded to the surface, and thus more easily moved by the STM tip. This effect may occur because the silver atoms bond to specific atomic sites on the graphite surface ( $\beta$ sites) and these sites have a separation which is different from the preferred separation of silver atoms in the bulk crystal. A small silver island can accommodate this strain, and we have identified that the majority of atoms in small silver clusters sit on the $B$ sites (11). But as the island grows it is likely that the atoms relax towards their preferred bulk positions and the strength of the bond 
with the surface decreases. We expect this behaviour to be mirrored in the case of gold clusters, and in the STM image (Figure 1) a build up of larger gold particles in the bottom right hand corner of the scan is observed. We believe that these are larger clusters that have been moved by the scanning tip and deposited at the end of a scan. With a change in the bias voltage applied to the tip, such that the tip scans further away from the surface, we have been able to minimize or eliminate this effect.

\section{APPLICATIONS}

We have seen that the presence of the STM tip can significantly perturb a surface. Indeed, the STM has been used by Eigler and colleagues (12) to move individual atoms on a $\mathrm{Ni}(110)$ surface by dragging them from one atomic position to another. By this procedure it was possible to write the 'IBM' logo on a surface in xenon atoms. Although this may seem the ultimate way to build nanostructures, it is an extremely time consuming process taking many hours to complete. Moreover, the controlled manipulation of clusters would also be a very time consuming process. However, the self organization of atoms on a patterned surface, e.g., on an artificially produced step structure, should be much quicker and could eventually represent a new route to the large scale production of nanometre size devices. Gold is chemically inert and represents a favourable choice of material for the manufacture of electrical components; by contrast, silver forms an inert sulfide surface layer on exposure to air (13).

As discussed above, we can now produce long wires of gold and silver clusters (10) along step edges of graphite, and in the future one might imagine gold wires like these as connections between components in a nanoscale electrical circuit. One of our current research programmes is concerned with the assembly of wires and other nanoscale electronic components from size selected noble metal clusters deposited onto a pre-patterned surface.

In integrated circuit manufacture the current state of the art minimum line width produced by optical lithography techniques is a few hundred nanometres. In the next few years deep-ultra-violet lithography will probably be used (14) which is thought capable of producing minimum line widths towards $100 \mathrm{~nm}$. Electron beam lithography can go further giving line widths of around $10 \mathrm{~nm}$. So it can be seen that the dimensions of the gold wires which could be assembled from clusters are more than competitive with the limits of resolution of these other technologies.

Over the last few years atomic clusters have begun to show rich potential for exploitation in a number of areas. The fact that their properties are size dependent suggests that materials could be specifically tailored for a wide range of applications areas from electronics and computing to sensors and catalysis (15). The present work, which illustrates the potential of gold clusters as building blocks for nanoscale circuitry, is very much concerned with the scientific basis for these technological opportunities, and much work still needs to be done at this level. But it is already clear that gold has an important place in the expanding world of science and technology on the nanometre scale.

\section{ACKNOWLEDGEMENTS}

We thank EPSRC for financial support of this work, and we are grateful to Dr N. Berovic for access to the Burleigh scanning tunnelling microscope.

\section{ABOUT THE AUTHORS}

Gareth Francis is currently studying for his doctorate at the Nanoscale Physics Research Laboratory in the School of Physics and Space Research at the University of Birmingham.

Richard Palmer is Professor of Experimental Physics and Head of the Nanoscale Physics Research Laboratory, which was formally opened on May 8th 1996. The goal of the new laboratory is to advance the frontiers of the physics, chemistry and technology of nanometre-scale structures, devices and processes. Professor Palmer has been awarded the 1996 Charles Vernon Boys Medal and Prize by the Institute of Physics.

\section{REFERENCES}

1 M. Moskovits, Annu. Rev. Phys. Chem., 1991, 42, 465

2 G.K. Wertheim, Phase Transitions, 1990, 24-26, 203

3 W. A. De Heer, W.D. Knight, M.Y. Chou and M.L. Cohen, in Solid State Physics, ed. H. Ehrenreich and D. Turnbull, Academic Press Inc, 1987, 40, 93 
4 M.F. Jarrold, in 'Clusters of Atoms and Molecules I', ed. H. Haberland, Springer Series in Chemical Physics, Springer-Verlag, 1995, 52, 315

5 L.D. Marks and D.J. Smith, Nature, 1983, 303, 316

6 G. Binnig, H. Rohrer, Ch. Gerber and E. Weibel, Phys. Rev. Lett., 1982, 49, 57

H.J. Mamin, S. Chiang, H. Birk, P.H. Guethner and D. Rugar, J. Vac. Sci. Technol., 1991, B9, 1398

8 M. Dorogi, J. Gomez, R. Osifchin, R.P. Andres and R. Reifenberger, Phys. Rev. B, 1995, 52, 9071

9 D.G. Duff and A. Baiker, 'Studies in Surface Science and Catalysis', 1995, 91, 505
10 G.M. Francis, L. Kuipers, J.R.A. Cleaver and R.E. Palmer, J. Appl. Phys., 1996, 79, 2942

1 G.M. Francis, I.M. Goldby, L. Kuipers, B. von Issendorff and R.E. Palmer, J. Chem. Soc. Dalton Trans., 1996, 665

12 D.M. Eigler and E.K. Schweizer, Nature, 1990, 344, 524

13 N.V. Sidgwick, 'The Chemical Elements and their Compounds', Vol. 1, Oxford University Press, 1952, p.119

14 G. Kamarinos and P. Felix, J. Phys. D: Appl. Phys., 1996, 29, 487

15 S.N. Khanna and P. Jena, Phys. Rev. Lett., 1992, 69, 1664 